\title{
Hypomineralisation or hypoplasia?
}

\section{IN BRIEF}

- Provides general dental practitioners with an overview of the background and aetiology of enamel hypomineralisation and hypoplasia

- Outlines the different characteristics and clinical variabilities between hypomineralisation and hypoplasia

- Provides an understanding of how to diagnose hypomineralisation and hypoplasia and guide management

\begin{abstract}
Enamel hypomineralisation is a qualitative defect, with reduced mineralisation resulting in discoloured enamel in a tooth of normal shape and size. Because the enamel is weaker, teeth can undergo post eruptive breakdown, resulting in missing enamel. Enamel hypoplasia is a quantitative defect of the enamel presenting as pits, grooves, missing enamel or smaller teeth. It can sometimes be difficult to differentiate between the two. In this review paper, we aim to explain the importance of differentiating between the two conditions, and how to manage patients presenting with enamel defects.
\end{abstract}

\section{HOW DOES ENAMEL FORM?}

Enamel is produced by specialised end-differentiated cells known as ameloblasts. ${ }^{1}$ The formation of enamel can be separated into initial stages which involve secretion of matrix proteins such as amelogenin, ameloblastin and enamelin, and later stages of mineralization and maturation. ${ }^{1}$

Tooth enamel is unique due to its high mineral content. It is composed of highly organised, tightly packed hydroxyapatite crystallites that comprise $87 \%$ of its volume and $95 \%$ of its weight, with the remainder comprising of organic matrix and water. ${ }^{1}$ This pattern of organisation and mineralisation gives enamel its significant physical properties, making it the hardest tissue in the body. ${ }^{1}$

Developmental defects of enamel are not uncommon, both in the primary and permanent dentitions. ${ }^{1}$ Environmental and/or genetic factors that interfere with tooth formation are thought to be responsible for both hypomineralisation and hypoplasia., ${ }^{1,2}$ If a disturbance occurs during the secretion phase, the enamel defect is called hypoplasia. If it occurs during the mineralisation or maturation phase, it is called hypomineralisation. Often the cause is difficult to determine.

The psychological impact of enamel defects in children should not be underestimated, and it is important to be aware of the impact of being teased for having different looking teeth. 
Dental appearance influences how individuals are perceived by others and young people make negative psychosocial judgements on the basis of enamel appearance. ${ }^{3}$

\section{WHAT IS HYPOMINERALISATION AND HYPOPLASIA?}

Hypomineralisation:

A reduced quality of enamel is termed hypomineralisation. The enamel is of normal thickness, but not fully mineralised. Enamel hypomineralisation can be seen due to differences in translucency; enamel can appear opaque, creamy white or have yellow/brown discolorations. Opacities may be diffuse and/or demarcated. Figure 1 demonstrates the appearance of generalised diffuse opacities.

Hypomineralised defects can be localised or generalised. Local hypomineralised defects can be caused by trauma or infection to the primary tooth, or radiation. Generalised hypomineralised defects are often caused by systemic, environmental or genetic factors as described in Table 1. Systemic / environmental attacks that occur in a specific window of time, will only affect teeth mineralising during that period, as seen in chronological hypomineralisation. Fluorosis can cause enamel hypomineralisation which can be either localised or generalised and presents as diffuse, linear or patchy white opacities without a clear boundary. Patients with Amelogenisis Imperfecta can present with hypomineralised or hypoplastic defects which tend to be generalised.

The most common hypomineralisation defect seen in children is Molar incisor hypomineralisation $(\mathrm{MIH})$, which affects the first permanent molar teeth and often the permanent incisors. ${ }^{2,4}$ The pooled prevalence of $\mathrm{MIH}$ is $14.2 \%$ globally. ${ }^{5}$ There is an association between $\mathrm{MIH}$ and hypomineralisation affecting the second primary molars. ${ }^{6} \mathrm{MIH}$ can vary in presentation, from small areas of discolouration to extensive post eruptive breakdown (PEB), which can occur rapidly and give rise to sensitivity and pain, caries and be difficult to restore. ${ }^{4}$ Enamel missing due to PEB tends to leave rough, uneven border between the affected and unaffected enamel. In comparison enamel missing due to hypoplasia it often smooth with rounded edges. The severity of hypomineralisation is usually less in affected incisors compared to affected molars, but aesthetic concerns may be an issue.

Hypoplasia:

Enamel hypoplasia is a reduced quantity of enamel which results in small or irregular shaped teeth, which may be pitted, thinner or smaller in size (Figure 2). Localised hypoplastic defects can be caused by trauma or infection in the primary tooth. Generalised hypoplastic defects can also be due to systemic, environmental and genetic factors.

The most common cause of chronological hypoplasia seen in children is due to vitamin D deficiency. ${ }^{1}$ Chronological hypoplasia is a generalised form of hypoplasia, with a characteristic presentation of symmetrical defects with a chronological pattern, with only parts of the teeth developing (enamel secretion) at the time of the insult affected. In chronological hypoplasia, 
the border is smooth and rounded, as opposed to the rough border in PEB, and only affects a portion of the crown, as shown in figures 2 and 3.

It is important to be aware of the different aetiological factors for enamel hypomineralisation and hypoplasia to aid diagnosis, as highlighted in Table 1.

Table 1 - Aetiology of enamel hypoplasia and hypomineralisation 1,2,4

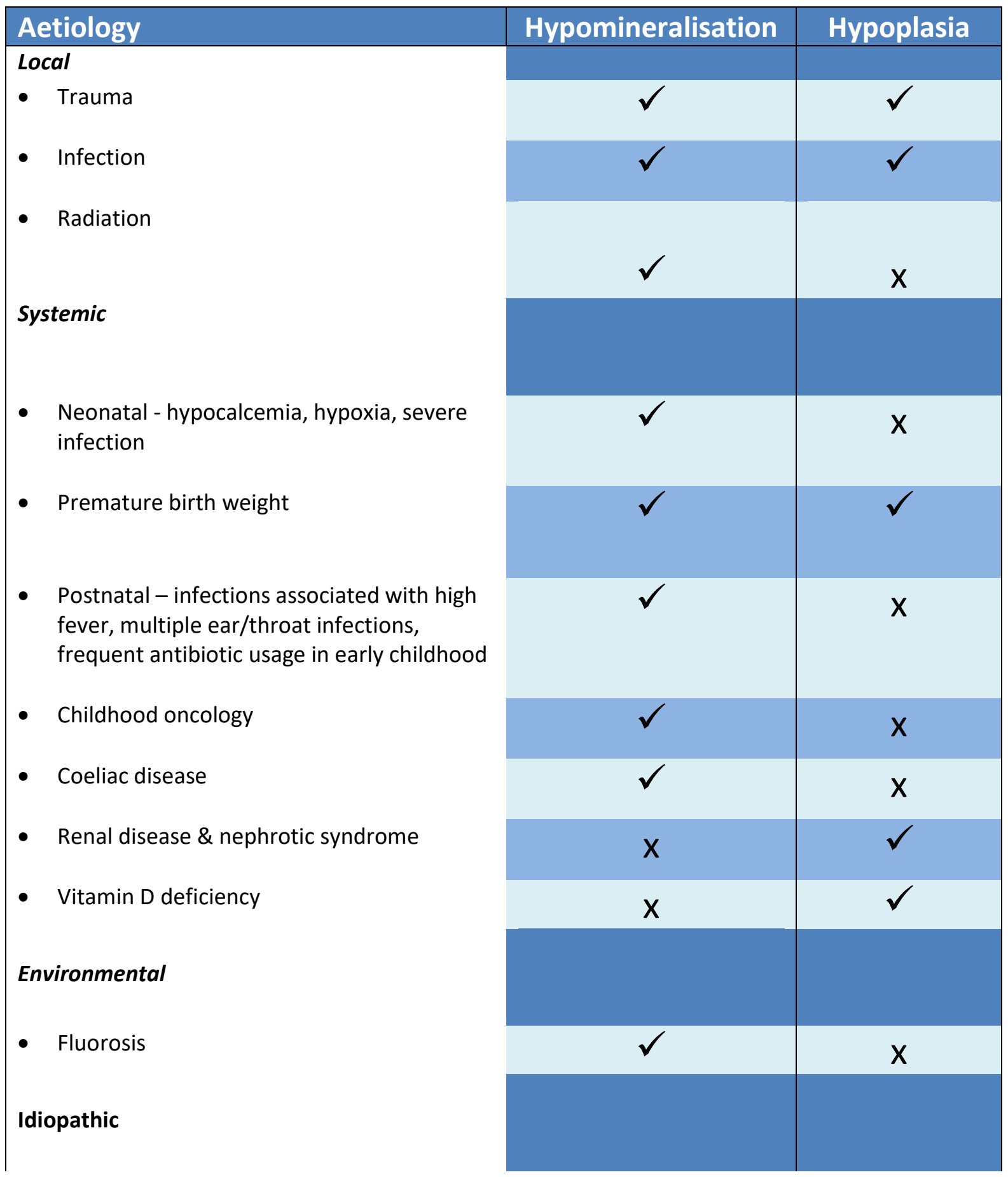


- Molar Incisor Hypomineralisation (MIH)

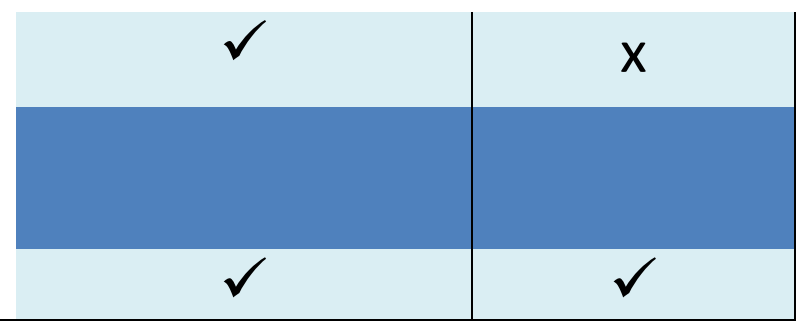

Hypomineralised and hypoplastic teeth show a spectrum of clinical variability. To help recognise the difference between the two, the most common characteristics are outlined in Table 2.

\begin{tabular}{|l|c|c|}
\hline CHARACTERISTIC & HYPOPLASIA & HYPOMINERALISATION \\
\hline Pitted Enamel & $\checkmark$ & X \\
\hline White to yellow Opacities & X & $\checkmark$ \\
\hline Hard Enamel & $\checkmark$ & X \\
\hline Soft, porous enamel & $\mathrm{X}$ & $\checkmark$ \\
\hline Poor quality enamel & $\mathrm{X}$ & $\checkmark$ \\
\hline Reduced amount of enamel & $\checkmark$ & $\mathrm{X}$ \\
\hline Bonding affected & $\mathrm{x}$ & $\checkmark$ \\
\hline
\end{tabular}

Table 2 Characteristics of enamel hypoplasia and hypomineralisation 1,2,4,7

\section{HOW DOES THE MANAGEMENT DIFFER?}

Understanding the properties of hypomineralised and hypoplastic teeth is fundamental in improving restorative outcomes.

Patients often complain of sensitivity with both hypomineralised and hypoplastic teeth. Simple measures to manage sensitivity include advising patients to use warm water during toothbrushing, using sensitive fluoride toothpaste, professional application of topical fluoride varnish or provision of Tooth Mousse ${ }^{\circledR}$. 
Figures 3 and 4 summarise the treatment options for anterior and posterior teeth.

\section{(i) Hypomineralised}

Hypomineralised teeth are more prone to dental caries, post eruptive break down and often require complex restorations therefore management is challenging. ${ }^{1}$

\section{$\underline{\text { Anterior teeth }}$}

It is important to consider that it is sometimes difficult to achieve direct adhesion with composite resin to teeth with poorly mineralised enamel.1,7,8 In addition to this, hypomineralised teeth are often a normal shape and size, therefore tooth preparation will be required to create a normal emergence profile and tooth morphology. Therefore, conservative management including tooth whitening and microabrasion should always be considered initially as a less invasive treatment modality (Figure 6a, 6b). Another microinvasive alternative is resin infiltration. It can be used to treat white spots in vestibular areas. The principle is that air and water entrapments in the tooth have a lower refractive index than intact tooth structure which leads to unesthetic discolorations. Resin infiltration balances out this difference and the appearance blends in with the healthy enamel masking the whitish appearance of the lesions.

\section{Posterior teeth}

It is important to assess the prognosis of affected molar teeth, when deciding the most appropriate treatment. If a permanent molar is minimally affected, with no symptoms or post eruptive breakdown, simple preventive measures such as fluoride varnish and fissure sealants are suitable. If the patient is un-cooperative or has sensitivity glass ionomer cement (GIC) fissure sealants can be considered. ${ }^{9}$

It is important to assess the long term prognosis of the first permanent molars in MIH before ten years of age. Severely hypomineralised teeth of poor prognosis, should ideally be managed by a multidisciplinary team, including a specialist paediatric dentist and orthodontist, to consider planned extractions if appropriate. Molars can be stabilised with a preformed metal crown (PMC) or resin modified GIC. The complete coverage of a PMC reduces sensitivity, prevents cusp fracture and helps maintain occlusal vertical dimension and crown height ${ }^{1}$ (Figure 7). An additional benefit is that the technique is conservative with minimal removal of tooth structure. In younger patients it can be used as an interim restoration whilst waiting for optimal timing of extractions of poor prognosis first permanent molars. If indirect restorations such as onlays or crowns are a treatment option, the extent of sound enamel should be carefully considered as this can have a direct effect on bonding. Enamel deproteinization with $5 \% \mathrm{NaOCl}$ before adhesive application procedure has been suggested to enhance bonding performance of resin dental adhesives to hypomineralized 
enamel. ${ }^{10}$ If onlays are to be placed, gold/ nickel chromium/ cobalt chromium onlays are materials of choice as they require minimal preparation and are conservative to tooth tissue.

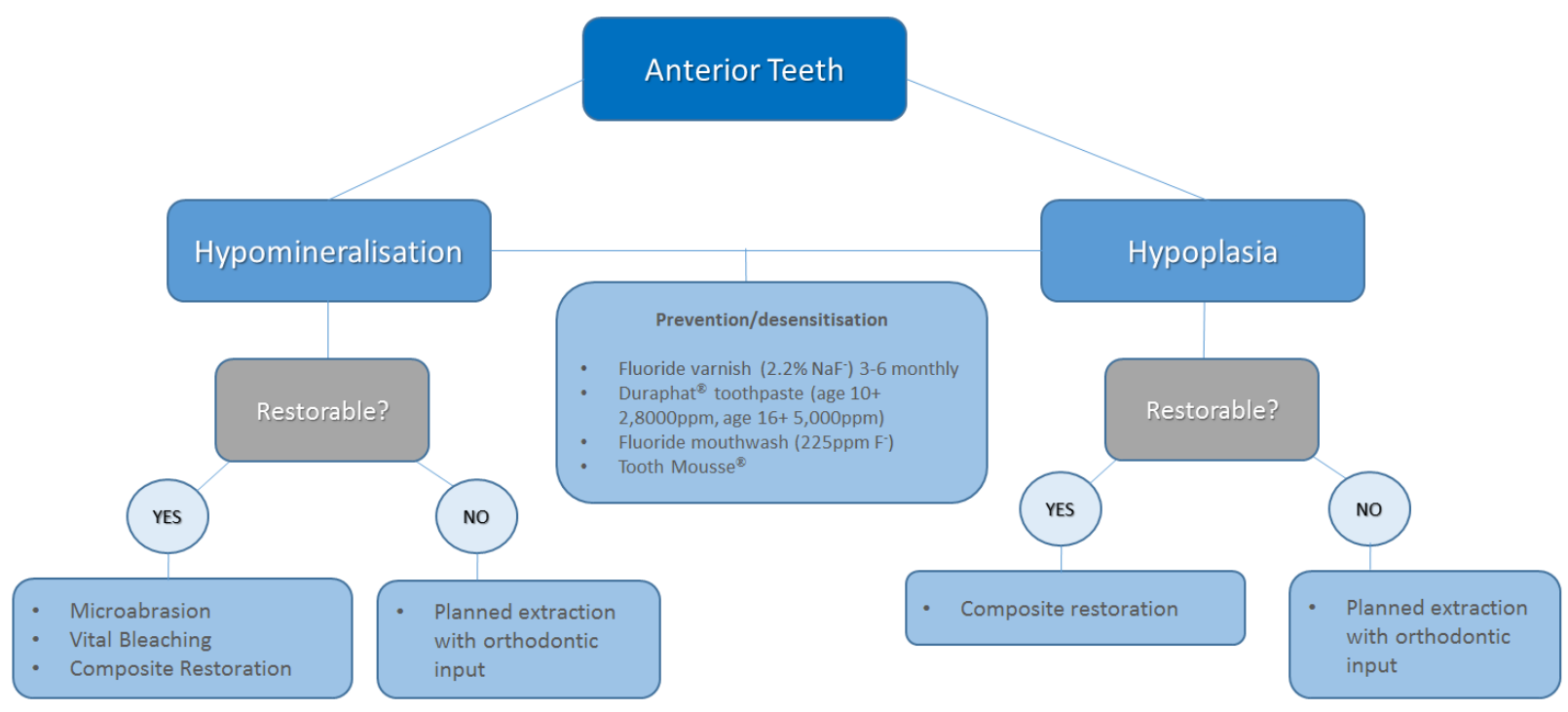

Fig. 4 Treatment options for anterior teeth $1,4,7,9$

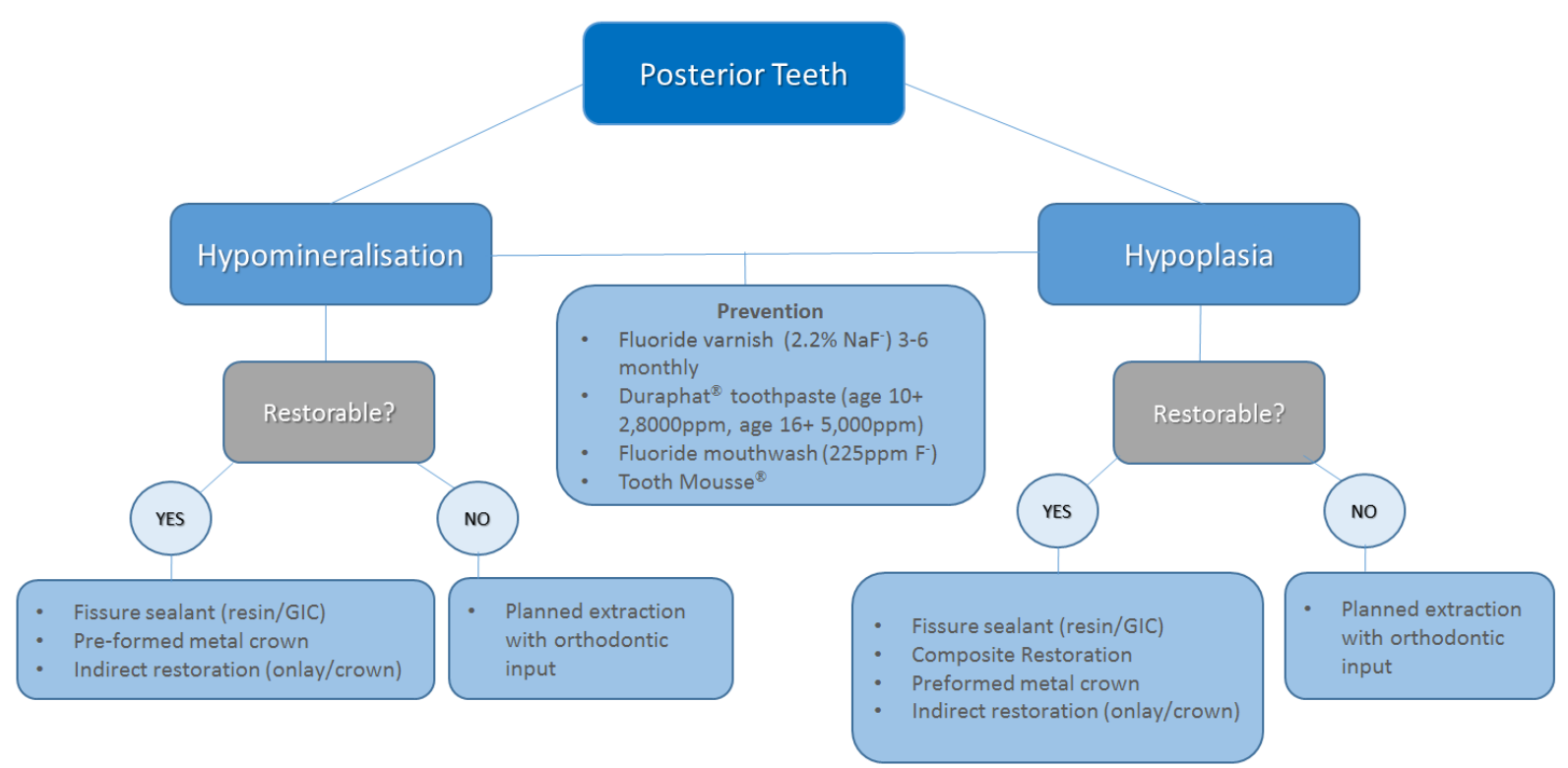

Fig. 5 Treatment options for posterior teeth ${ }^{1,4,7,9}$

(ii) Hypoplasia 
Hypoplastic teeth may present with pits or have a reduced thickness but the quality of the enamel is not altered, therefore bonding should be unaffected. However, there is limited evidence to support this at present. ${ }^{4}$ Options such as vital bleaching, microabrasion and resin infiltration are not usually appropriate, as the main issue is shape and not colour.

\section{Anterior teeth}

If the teeth are asymptomatic and the patient has no aesthetic concerns, then no treatment is necessarily indicated. However, hypoplastic teeth can appear smaller and be an aesthetic concern for the patient. The addition of direct composite restorations is straight forward, often no preparation is required to restore the tooth to its normal morphology.

\section{$\underline{\text { Posterior teeth }}$}

If posterior teeth are minimally affected fissure sealants or composite restorations are suitable. If the patient is un-cooperative or has extreme sensitivity, glass ionomer cement (GIC) fissure sealants can be considered. ${ }^{9}$ If the whole tooth surface is significantly affected preformed metal crowns, composite restorations or adhesive onlays can be considered.

Severely affected hypoplastic teeth of poor prognosis, should be managed by a multidisciplinary team and assessed by a specialist paediatric dentist and orthodontist to consider planned extractions if appropriate.

\section{CONCLUSION}

Correct diagnosis in patients presenting with enamel defects is crucial to indicate appropriate management. Preventative measures including fluoride treatments and fissure sealants can reduce sensitivity, and minimally invasive treatment options should be used where possible to preserve tooth structure. 


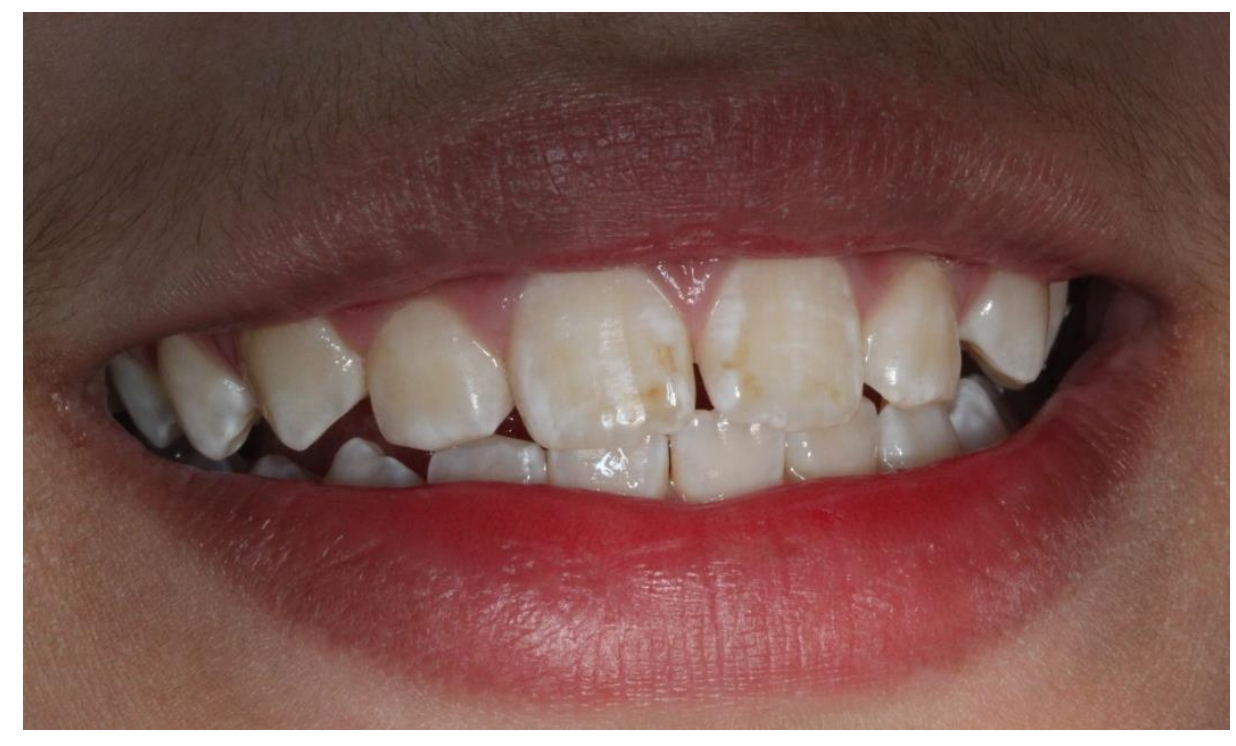

Fig. 1 Enamel hypomineralisation

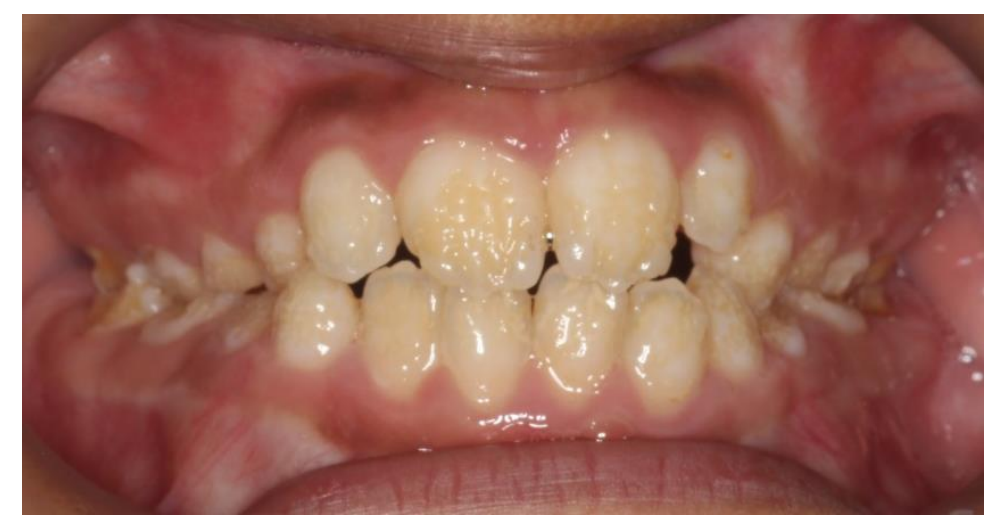

Fig. 2 Enamel Hypoplasia 


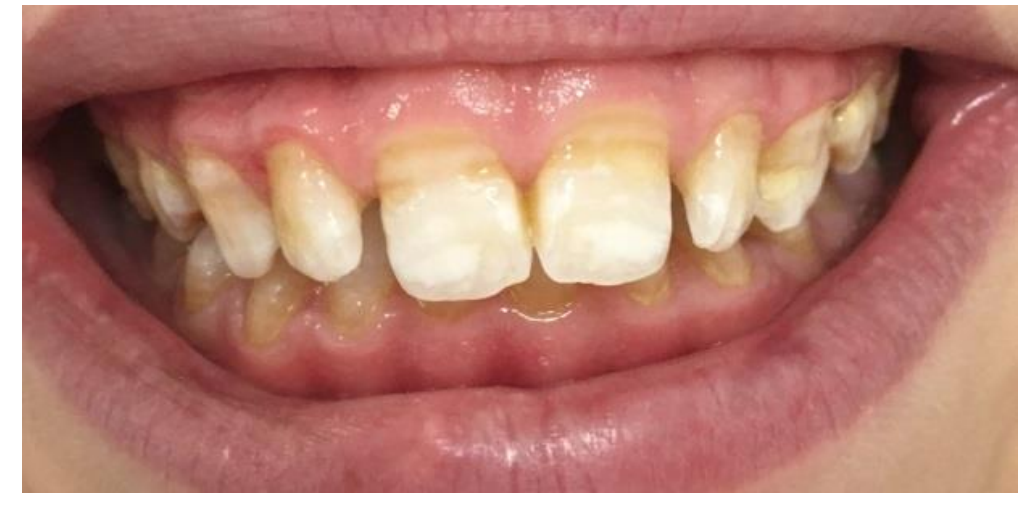

Fig. 3 Chronological hypoplasia

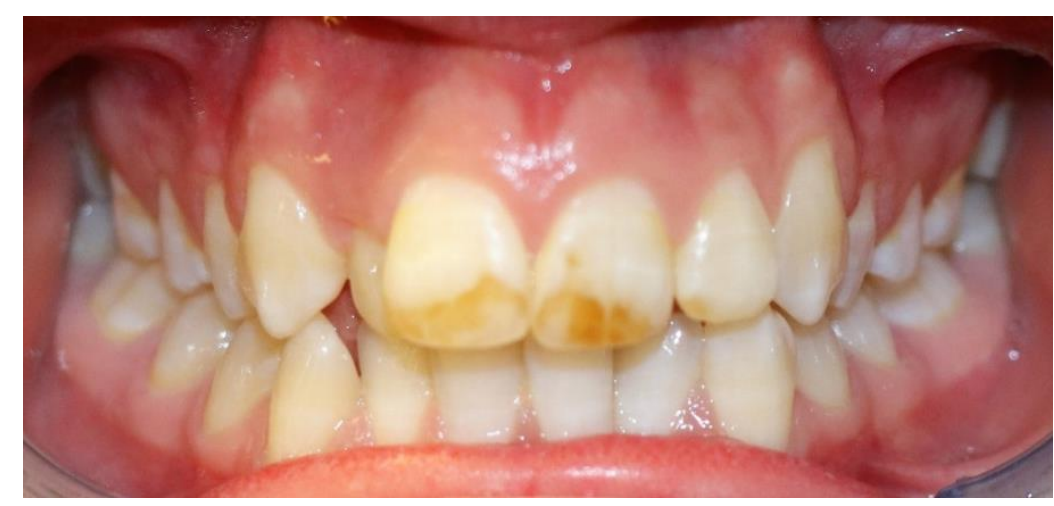

Fig. 6a Patient with molar incisor hypomineralisation prior to microabrasion and vital bleaching

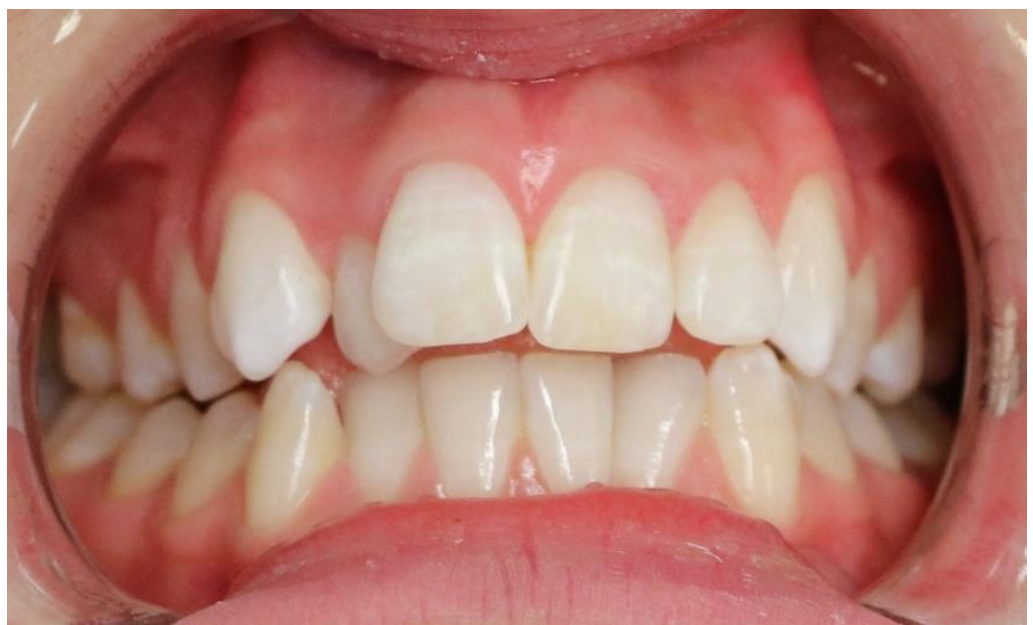

Fig. 6b Post micro abrasion and vital bleaching 


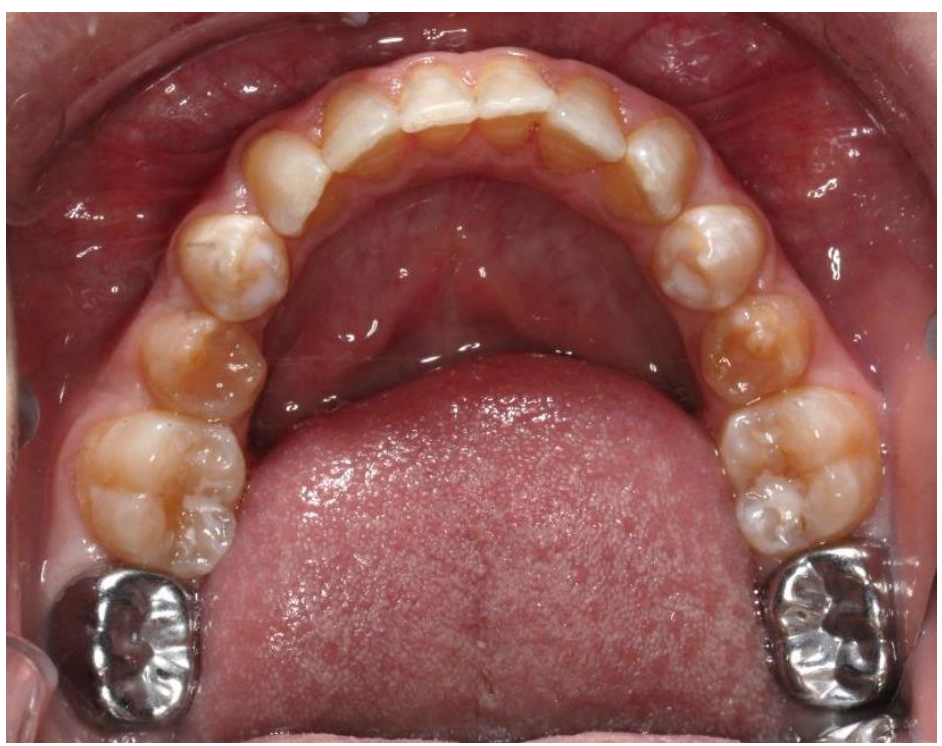

Fig. 7 Preformed metal crows placed on the LL7, LR7 on a patient with chronological hypoplasia

References:

1. Seow WK. Developmental defects of enamel and dentine: challenges for basic science research and clinical management. Australian dental journal. 2014; 1(59): 143-54.

2. Beentjes VE, Weerheijm KL, Groen HJ. Factors involved in the aetiology of molarincisor hypomineralisation (MIH). European Journal of Paediatric Dentistry. 2002; 3:9-13.

3. Craig SA, Baker SR, Rodd HD. How do children view other children who have visible enamel defects? International Journal of Paediatric Dentistry. 2015; 25: 399-408.

4. Weerheijm KL, Jälevik B, Alaluusua S. Molar-incisor hypomineralisation. Caries research. 2001;35(5):390-1.

5. Zhao D, Dong $B, Y u$ D, Ren $Q$, Sun $Y$. The prevalence of molar incisor hypomineralization: evidence from 70 studies. International Journal of Paediatric Dentistry. 2018;28(2):170-179. 
6. Elfrink ME, Cate JM, Jaddoe VW, Hofman A, Moll HA, Veerkamp JS. Deciduous molar hypomineralization and molar incisor hypomineralization. Journal of Dental Research. 2012 91(6):551-5.

7. Mahoney E, Ismail FS, Kilpatrick N, Swain M. Mechanical properties across hypomineralized/hypoplastic enamel of first permanent molar teeth. European journal of oral sciences. 2004;112(6): 497-502.

8. William V, Burrow MF, Palamara JE, Messer LB. Microshear bond strength of resin composite to teeth affected by molar hypomineralisation using 2 adhesive systems. Pediatric dentistry. 2006;28(3): 233-41.

9. Scottish Clinical Dental Effectiveness Programme (SCDEP). Prevention and Management of Dental Caries in 2018; www. http://www.sdcep.org.uk/wpcontent/uploads/2018/05/SDCEP-Prevention-and-Management-of-Dental-Caries-inChildren-2nd-Edition.pdf. Accessed 3rd March 2019.

10. Ekambaram M, Yiu CK. International Journal of Adhesion and Adhesives Bonding to hypomineralized enamel - A systematic review. 2016; 69:27-32. 